\title{
Broadband Microstrip Bandpass Filter Based on Open Complementary Split Ring Resonators
}

\author{
P. Vélez, J. Naqui, M. Durán-Sindreu, J. Bonache, and F. Martín \\ GEMMA/CIMITEC, Departament d'Enginyeria Electrònica, Universitat Autònoma de Barcelona, \\ Barcelona 08193 Bellaterra, Spain \\ Correspondence should be addressed to P.Vélez, paris.velez@uab.cat
}

Received 6 September 2012; Accepted 26 October 2012

Academic Editor: Alejandro Lucas Borja

Copyright ( 2012 P. Vélez et al. This is an open access article distributed under the Creative Commons Attribution License, which permits unrestricted use, distribution, and reproduction in any medium, provided the original work is properly cited.

Broadband bandpass filters based on open complementary split ring resonators (OCSRRs) coupled through admittance inverters, and implemented in microstrip technology, are reported. As compared to other broadband filters based on open split ring resonators (OSRRs), ground plane etching is not necessary in the proposed filters. The selectivity of the filters at the upper transition band is improved thanks to the presence of a controllable transmission zero. To demonstrate the potential of this approach, two illustrative prototype devices have been designed and fabricated.

\section{Introduction}

Metamaterials have been a subject of increasing interest in the last decade. Soon after the synthesis of the first lefthanded metamaterial based on a combination of metallic posts and split ring resonators (SRRs) in 2000 [1], the first works devoted to extend the properties and concepts of metamaterials to the design of microwave circuits in planar technology came into the scene [2-5]. In particular, it was demonstrated in [5] that a coplanar waveguide (CPW) transmission line loaded with SRRs [6] and shunt metallic strips exhibits a bandpass functionality with backward (or left-handed) wave propagation in the first allowed band. The CPW-based structure reported in [5] was the planar analog of the bulk structure reported in [1] and was the seed for further design of microwave filters based on SRRs, on their complementary counterparts (the complementary split ring resonator-CSRR [7]), or on other metamaterial resonators [8-18].

The filters reported in [8-18] are based on closed resonators (SRRs or CSRRs) coupled to a host line. However, filters based on electrically small open resonators, like the open split ring resonator (OSRR) [19], or the open complementary split ring resonator (OCSRR), [20] have also been reported [21-25]. As compared to SRRs/CSRRs, the open resonators (OSRRs/OCSRRs) are electrically smaller by a factor of two $[19,20,26]$. Moreover, OSRRs and OCSRR are intrinsically wideband resonators (the reason for that is that, as compared to SRRs or CSRRs, OSRRs and OCSRRs exhibit high $C / L$ and $L / C$ ratios, resp., due to their topology, and this favors broadband responses); therefore, these open resonators are of interest for the implementation of moderate and wideband bandpass filters. It is remarkable that order3 [27], order-5 [28], and order-7 [29] Chebyshev bandpass filters based on a combination of OSRRs and OCSRRs have been designed and fabricated. In such filters, the OSRRs and the OCSRRs are described by means of series and parallel resonators, respectively. Despite the fact that some parasitics must be introduced in the model in order to adequately describe the resonators loading a host line, the effects of these parasitics are not meaningful, and the filters exhibit the intended Chebyshev responses to a very good approximation.

In this paper, OCSRRs are used for the implementation of broadband bandpass filters in microstrip technology. The filters are similar to those filters reported in [20], that is, shunt connected OCSRRs coupled through admittance inverters. However, the filters in [20] are implemented in CPW technology, whereas the filters proposed in this paper are implemented in microstrip technology. As compared 


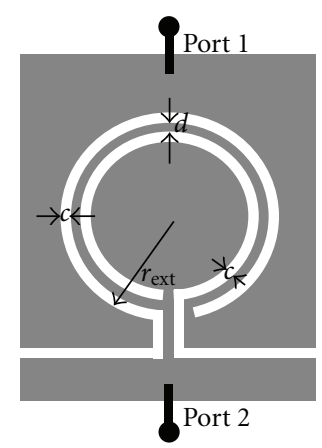

(a)

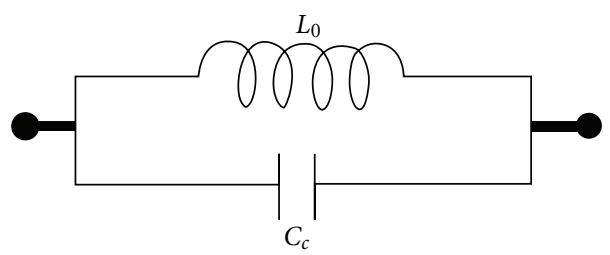

(b)

FIGURE 1: Typical topology of the OCSRR (a) and equivalent circuit model (b). The two terminals—ports—of the open resonator are indicated.

to the filters based on OSRRs and also implemented in microstrip technology, the advantage of our approach is that ground plane etching is not necessary. Moreover, the new proposed filters exhibit a transmission zero above the passband of interest that can be used to improve the filter selectivity above the pass band.

The paper is organized as follows. In Section 2, we will review the OCSRR for completeness, since this resonator is the key building block of the proposed filters. The design of the proposed broadband filters, including the simulation and measurement of the filter responses, are reported in Section 3. Finally, the main conclusions are highlighted in Section 4.

\section{Open Complementary Split Ring Resonators (OCSRRs)}

The OCSRR is the open version of the CSRR. The typical topology and circuit model of the OCSRR is depicted in Figure 1. For the OCSRR, the particle terminals (or ports) are indicated in the figure. Between these ports, there is an electric short through the metal between the inner and outer slot rings forming the particle, but there is also capacitive connection through the capacitances across the slots. Thus, according to this, the circuit model of the particle is an open parallel resonant tank, as Figure 1(b) illustrates. The inductance of the particle, $L_{o}$, is the inductance of the metallic strip between the ring slots, and the capacitance, $C_{c}$, is identical to the capacitance of the CSRR, that is, the capacitance of a disk of radius $r_{o}-c / 2$ (where $r_{o}=r_{\text {ext }}-c-$ $d / 2$ is the averaged radius) surrounded by a metallic plane at a distance $c$ of its edge [30] (notice that this means that the capacitance is identical to that of a slot ring with slot width $c$ and average radius $r_{o}$, as it is justified in [30]). Since the inductance of the CSRR is $L_{o} / 4$ [30], it is expected that the resonance frequency of the OCSRR is one half the resonance frequency of the CSRR. The detailed calculation of $C_{c}$ is reported in [30], whereas $L_{o}$ is the inductance of the circular CPW structure that results from the OCSRR topology.

The previous model can be improved by considering the inductive effect of the metallic strip connecting the inner region of the particle and the metallic region in contact to port 2. This introduces an inductance in series with the parallel resonator, as reported in [29], and the effect of this inductance/strip is the presence of a short between ports 1 and 2 at a finite frequency (i.e., above the resonance frequency of the OCSRRs). In the bandpass filters based on OSRRs and OCSRRs reported by the authors, where the OCSRR is shunt connected to the host line, this short produces a transmission zero above the filter pass band. This improves the filter selectivity at the upper band and this strategy will be considered in the filters reported in this paper. However, in a first order approximation, the circuit of Figure 1(b) can be used for design purposes.

Let us now consider that the OCSRR is shunt connected to a microstrip transmission line. To effectively connect the resonator to ground, vias are required, as depicted in Figure 2(a). The structure is properly described by means of a shunt connected parallel resonant tank, as revealed by the reflection coefficient, represented in a Smith Chart in Figure 2(b) (notice that the reflection coefficient is always on the unit conductance circle, as expected from a reactive load in parallel to the $50 \Omega$ impedance of the port). Figure 2(c) indicates that the resonance frequency of the OCSRR is roughly $1.5 \mathrm{GHz}$, where total transmission (reflection zero) appears since the shunt branch is opened at this frequency. Notice that for this particular OCSRR topology, the strip connecting the inner region of the OCSRR and the host line is wide, and the inductive effect can be neglected, at least in the frequency region shown in the figure (the structure is adequately modeled by means of a parallel resonant tank in such region).

\section{Bandpass Filters Based on OCSRRs and Results}

The proposed bandpass filters are implemented by means of shunt resonators coupled through admittance inverters (see Figure 3), following the theory reported in [31]. The first prototype device filter is an order-3 Chebyshev band pass filter with central frequency $f_{o}=2 \mathrm{GHz}$, ripple of $0.01 \mathrm{~dB}$, and fractional bandwidth of $30 \%$. For these specifications, 


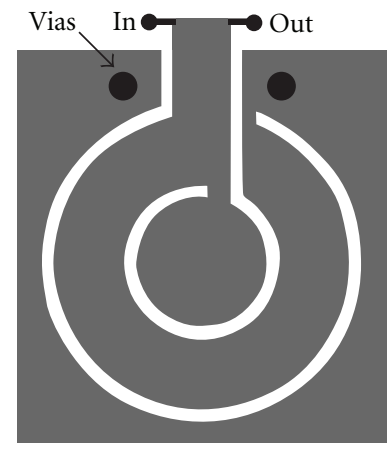

(a)

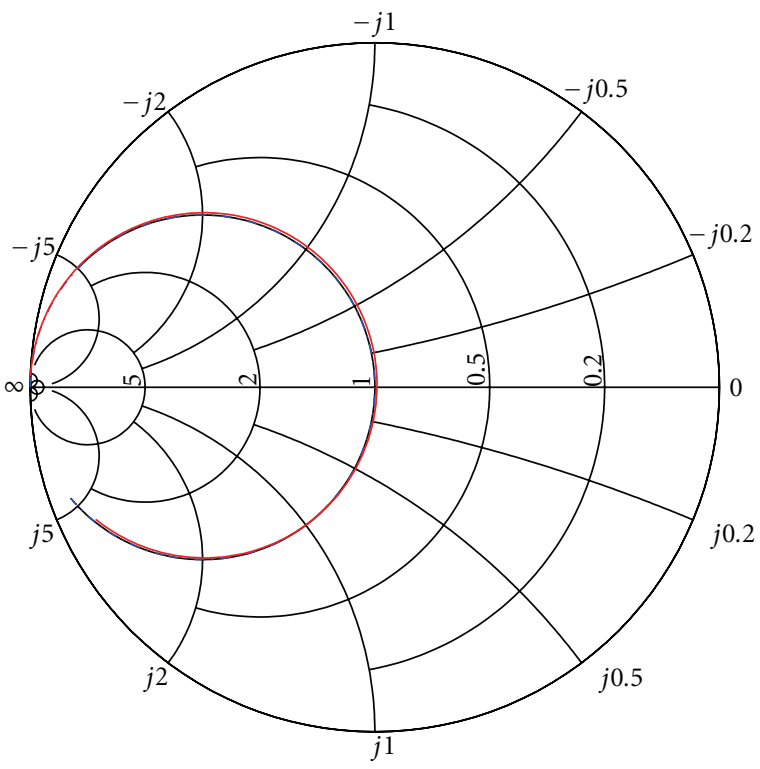

-... $S_{11}$ Circuit simulation

$-S_{11}$ EM simulation

(b)

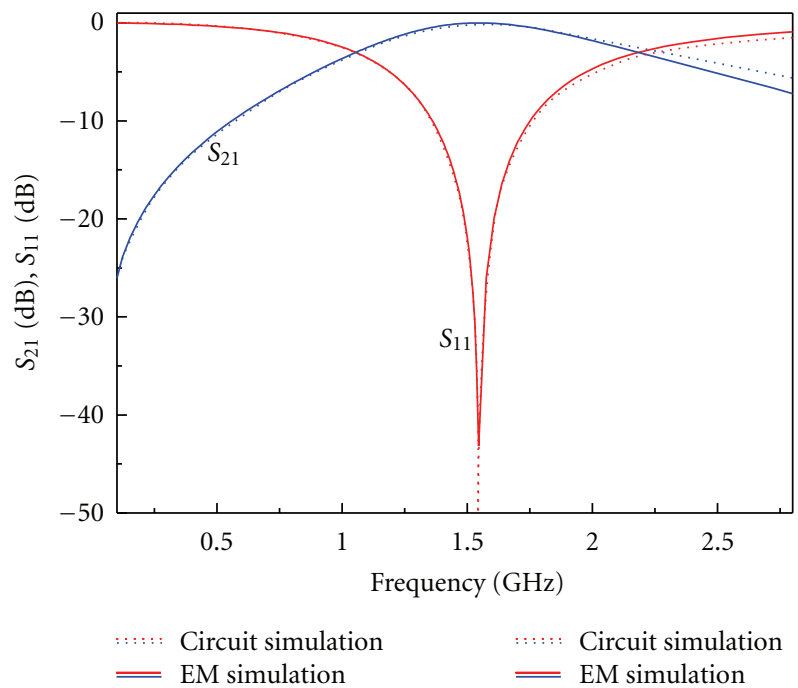

(c)

FIgure 2: Topology (a), Smith Chart (b), and frequency response (magnitude) (c) of the electromagnetic and circuit simulation of a shunt OCSRR in microstrip technology with $r_{\text {ext }}=2.7 \mathrm{~mm}, c=0.2 \mathrm{~mm}$, and $d=1.2 \mathrm{~mm}$. The considered substrate is the Rogers RO3010 with thickness $h=0.254 \mathrm{~mm}$ and dielectric constant $\varepsilon_{r}=10.2$. The element values of the shunt resonator in reference of Figure 1(b) are: $L_{o}=2.02 \mathrm{nH}$ and $C_{c}=5.25 \mathrm{pF}$.

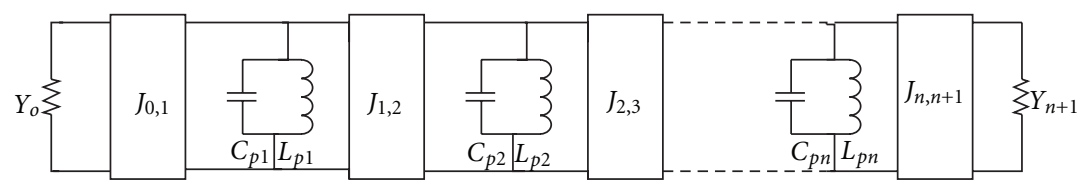

FIgURE 3: Bandpass filter network with admittance inverters and shunt LC resonators.

considering identical resonators with a value of inductance and capacitance of $L=1.4 \mathrm{nH}$ and $C=4.5 \mathrm{pF}$, respectively, the characteristic admittance of the inverters are found to be $J_{01}=J_{34}=0.023 \mathrm{~S}$, and $J_{12}=J_{23}=0.021 \mathrm{~S}$. The considered $L$ and $C$ values are those extracted from the layout of the isolated resonator, which gives the required central frequency. The inverters are implemented by means of $\lambda / 4$ lines ( $\lambda$ being the wavelength at the filter central frequency). The filter topology is depicted in Figure 4, where the main relevant dimensions are indicated (the filter has been fabricated following a standard photo/mask etching technique). The filter response, including the circuit simulation, the electromagnetic simulation (inferred by means of the Agilent Momentum commercial software), and the measured response (obtained by means of the Agilent PNA N5221A vector network analyzer) are depicted in Figure 5. 


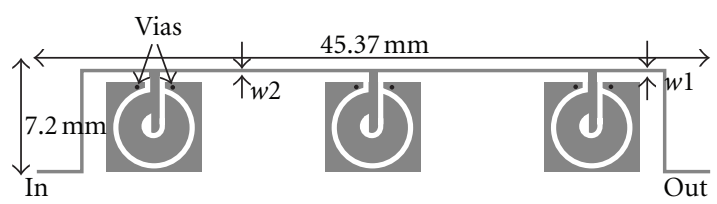

(a)

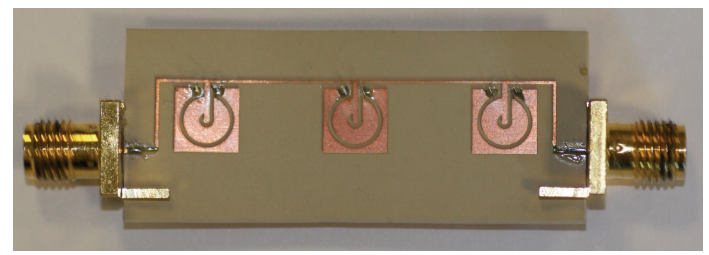

(b)

Figure 4: Topology (a) and photograph (b) of the proposed bandpass filter of the first example. The substrate is the Rogers $R O 3010$ with thickness $h=0.254 \mathrm{~mm}$ and dielectric constant $\varepsilon_{r}=10.2$. For the OCSRRs, $r_{\mathrm{ext}}=2.7 \mathrm{~mm}, c=0.37 \mathrm{~mm}$, and $d=1.63 \mathrm{~mm}$. The width of the transmission line inverters is $w 1=$ $0.33 \mathrm{~mm}$ for the external ones and $w 2=0.28 \mathrm{~mm}$ for the internal ones.

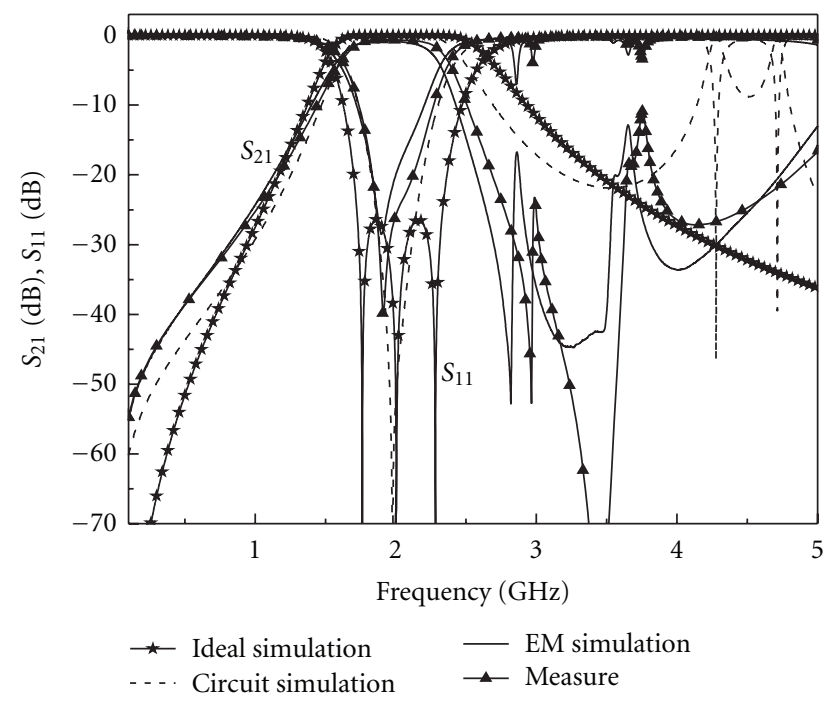

Figure 5: Frequency response of the filter of Figure 4. The circuit simulation has been achieved by means of Agilent ADS, considering lumped resonators coupled through $90^{\circ}$ at the central frequency.

The agreement between the electromagnetic simulation and measurement is remarkable. Notice that the measured filter selectivity at the upper edge of the band is much better than that obtained by the circuit simulation. The reason is the presence of the transmission zero above the passband, tailored by means of the width of the connecting strip between the inner region of the OCSRRs and the host line. Since the considered ripple is so small, the filter exhibits a quasi-Butterworth response, with a single reflection zero in the pass band. Filter performance is good, with a measured insertion loss smaller than $1.7 \mathrm{~dB}$ and return loss better than $-10 \mathrm{~dB}$ between $1.68 \mathrm{GHz}$ and $2.21 \mathrm{GHz}$. The filter upper

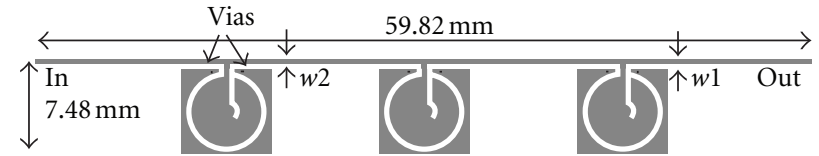

(a)

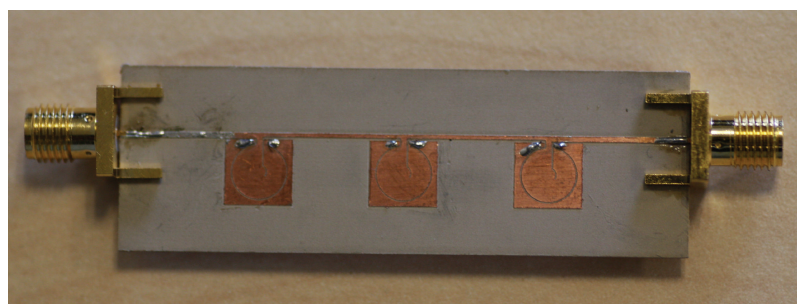

(b)

Figure 6: Topology (a) and photograph (b) of the proposed bandpass filter of the second example. The substrate is the Rogers $R O 3010$ with thickness $h=0.635 \mathrm{~mm}$ and dielectric constant $\varepsilon_{r}=$ 10.2. For the OCSRRs, $r_{\mathrm{ext}}=3 \mathrm{~mm}, c=0.16 \mathrm{~mm}$, and $d=2 \mathrm{~mm}$. The width of the transmission line inverters is $w 1=0.503 \mathrm{~mm}$ for the external ones and $w 2=0.335 \mathrm{~mm}$ for the internal ones.

stop band reveals that rejection is better than $-10 \mathrm{~dB}$ up to at least $5 \mathrm{GHz}$.

Notice that the filter (measured) bandwidth is smaller than the bandwidth of the ideal response since the inverters strictly exhibit their functionality at the central filter frequency. This aspect is well known and can be solved by considering a larger nominal bandwidth. However, this is not relevant for our purposes, since the main aim is to demonstrate the potential of OCSRRs for the design of broadband microstrip filters based on admittance inverters, as an alternative to OSRR-based microstrip filters.

The second example is an order- 3 Chebyshev band pass filter with central frequency $f_{o}=2 \mathrm{GHz}$, ripple of $0.1 \mathrm{~dB}$, and fractional bandwidth of $30 \%$. For these specifications, $L=$ $1.4 \mathrm{nH}$ and $C=4.5 \mathrm{pF}$, and the characteristic impedance of the inverters is $J_{01}=J_{34}=0.0184 \mathrm{~S}$, and $J_{12}=J_{23}=0.0156 \mathrm{~S}$. The topology and photograph of this filter are shown in Figure 6 and the frequency response in Figure 7. In this case, the pass band exhibits three reflection zeros and the return loss is better than $-20 \mathrm{~dB}$ between $1.77 \mathrm{GHz}$ and $2.25 \mathrm{GHz}$. Insertion loss is smaller than $0.9 \mathrm{~dB}$ within this frequency interval.

\section{Conclusions}

In conclusion, novel broadband microstrip bandpass filters based on OCSRRs coupled through admittance inverters have been proposed. The reported filters represent a significant progress as compared to other microstrip filters based on OSRRs, where ground plane etching in the region occupied by the OSRRs is necessary. The two designed filters exhibit good performance, and filter selectivity has been improved by the transmission zero inherent to the OCSRRs. 

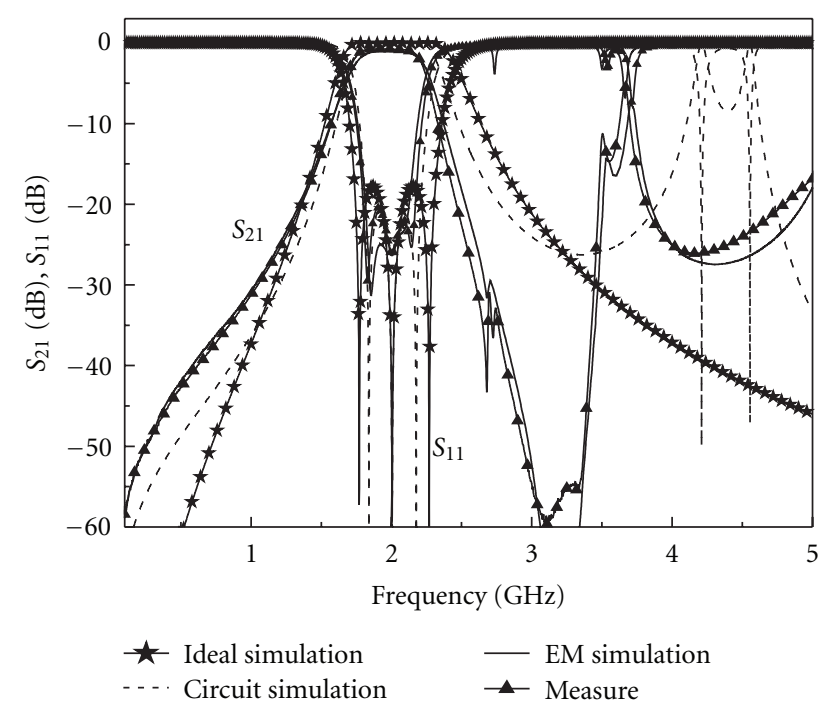

FIGURE 7: Frequency response of the filter of Figure 6.

\section{Acknowledgments}

This work has been supported by MICIIN-Spain (Contracts TEC2010-17512 and CSD2008-00066) and by Generalitat de Catalunya (Project 2009SGR-421).

\section{References}

[1] D. R. Smith, W. J. Padilla, D. C. Vier, S. C. Nemat-Nasser, and S. Schultz, "Composite medium with simultaneously negative permeability and permittivity," Physical Review Letters, vol. 84, no. 18, pp. 4184-4187, 2000.

[2] A. K. Iyer and G. V. Eleftheriades, "Negative refractive index metamaterials supporting 2-D waves," vol. 2, pp. 1067-1070.

[3] A. A. Oliner, "A periodic-structure negative-refractive-index medium without resonant elements," in Proceedings of the USNC-URSI National Radio Science Meeting, p. 41, San Antonio, Tex, USA, June 2002.

[4] C. Caloz and T. Itoh, "Application of the transmission line theory of left-handed ( $\mathrm{LH})$ materials to the realization of a microstrip 'LH line," in Proceedings of the IEEE Antennas and Propagation Society International Symposium, pp. 412-415, San Antonio, Tex, USA, June 2002.

[5] F. Martín, J. Bonache, F. Falcone, M. Sorolla, and R. Marqués, "Split ring resonator-based left-handed coplanar waveguide," Applied Physics Letters, vol. 83, no. 22, pp. 4652-4654, 2003.

[6] J. B. Pendry, A. J. Holden, D. J. Robbins, and W. J. Stewart, "Magnetism from conductors and enhanced nonlinear phenomena," IEEE Transactions on Microwave Theory and Techniques, vol. 47, no. 11, pp. 2075-2084, 1999.

[7] F. Falcone, T. Lopetegi, J. D. Baena, R. Marqués, F. Martín, and M. Sorolla, "Effective negative- $\varepsilon$ stopband microstrip lines based on complementary split ring resonators," IEEE Microwave and Wireless Components Letters, vol. 14, no. 6, pp. 280-282, 2004.

[8] F. Martín, F. Falcone, J. Bonache, R. Marqués, and M. Sorolla, "Miniaturized coplanar waveguide stop band filters based on multiple tuned split ring resonators," IEEE Microwave and Wireless Components Letters, vol. 13, no. 12, pp. 511-513, 2003.
[9] J. García-García, F. Martín, F. Falcone et al., "Spurious passband suppression in microstrip coupled line band pass filters by means of split ring resonators," IEEE Microwave and Wireless Components Letters, vol. 14, no. 9, pp. 416-418, 2004.

[10] J. Bonache, F. Martin, J. Garcia-Garcia, I. Gil, R. Marqués, and M. Sorolla, "Ultra wide band pass filters (UWBPF) based on complementary split rings resonators," Microwave and Optical Technology Letters, vol. 46, no. 3, pp. 283-286, 2005.

[11] J. Bonache, I. Gil, J. García-García, and F. Martín, "Novel microstrip bandpass filters based on complementary splitring resonators," IEEE Transactions on Microwave Theory and Techniques, vol. 54, no. 1, pp. 265-271, 2006.

[12] J. García-García, J. Bonache, I. Gil, F. Martín, M. Del Castillo Velázquez-Ahumada, and J. Martel, "Miniaturized microstrip and CPW filters using coupled metamaterial resonators," IEEE Transactions on Microwave Theory and Techniques, vol. 54, no. 6, pp. 2628-2635, 2006.

[13] P. Mondal, M. K. Mandal, A. Chaktabarty, and S. Sanyal, "Compact bandpass filters with wide controllable fractional bandwidth," IEEE Microwave and Wireless Components Letters, vol. 16, no. 10, pp. 540-542, 2006.

[14] M. Gil, J. Bonache, J. García-García, J. Martel, and F. Martín, "Composite right/left-handed metamaterial transmission lines based on complementary split-rings resonators and their applications to very wideband and compact filter design," IEEE Transactions on Microwave Theory and Techniques, vol. 55, no. 6, pp. 1296-1304, 2007.

[15] I. Gil, F. Martin, X. Rottenberg, and W. De Raedt, "Tunable stop-band filter at Q-band based on RF-MEMS metamaterials," Electronics Letters, vol. 43, no. 21, pp. 1153-1154, 2007.

[16] M. Gil, J. Bonache, and F. Martín, "Metamaterial filters: a review," Metamaterials, vol. 2, no. 4, pp. 186-197, 2008.

[17] A. L. Borja, J. Carbonell, V. E. Boria, J. Cascón, and D. Lippens, "Synthesis of compact and highly selective filters via metamaterial-inspired coplanar waveguide line technologies," IET Microwaves, Antennas and Propagation, vol. 4, no. 8, pp. 1098-1104, 2010.

[18] A. L. Borja, J. Carbonell, V. E. Boria, J. Cascon, and D. Lippens, "A 2\% bandwidth C-band filter using cascaded split ring resonators," IEEE Antennas and Wireless Propagation Letters, vol. 9, pp. 256-259, 2010.

[19] J. Martel, R. Marqués, F. Falcone et al., "A new LC series element for compact bandpass filter design," IEEE Microwave and Wireless Components Letters, vol. 14, no. 5, pp. 210-212, 2004.

[20] A. Vélez, F. Aznar, J. Bonache, M. C. Velázquez-Ahumada, J. Martel, and F. Martín, "Open complementary split ring resonators (OCSRRs) and their application to wideband cpw band pass filters," IEEE Microwave and Wireless Components Letters, vol. 19, no. 4, pp. 197-199, 2009.

[21] J. Martel, J. Bonache, R. Marqués, F. Martín, and F. Medina, "Design of wide-band semi-lumped bandpass filters using open split ring resonators," IEEE Microwave and Wireless Components Letters, vol. 17, no. 1, pp. 28-30, 2007.

[22] F. Aznar, A. Vélez, J. Bonache, J. Menés, and F. Martín, “Compact lowpass filters with very sharp transition bands based on open complementary split ring resonators," Electronics Letters, vol. 45, no. 6, pp. 316-317, 2009.

[23] F. Aznar, A. Vélez, M. Durán-Sindreu, J. Bonache, and F. Martin, "Elliptic-function CPW low-pass filters implemented by means of open complementary split ring resonators (OCSRRs)," IEEE Microwave and Wireless Components Letters, vol. 19, no. 11, pp. 689-691, 2009. 
[24] A. Vélez, F. Aznar, M. Durán-Sindreu, J. Bonache, and F. Martín, "Stop-band and band-pass filters in coplanar waveguide technology implemented by means of electrically small metamaterial-inspired open resonators," IET Microwaves, Antennas and Propagation, vol. 4, no. 6, pp. 712-716, 2010.

[25] A. Vélez, F. Aznar, M. Durán-Sindreu, J. Bonache, and F. Martín, "Tunable coplanar waveguide band-stop and bandpass filters based on open split ring resonators and open complementary split ring resonators," IET Microwaves, Antennas and Propagation, vol. 5, no. 3, pp. 277-281, 2011.

[26] F. Aznar, A. Vélez, M. Durán-Sindreu, J. Bonache, and F. Martín, "Open complementary split ring resonators: physics, modelling, and analysis," Microwave and Optical Technology Letters, vol. 52, no. 7, pp. 1520-1526, 2010.

[27] M. Durán-Sindreu, A. Vélez, F. Aznar, G. Sisó, J. Bonache, and F. Martín, "Applications of open split ring resonators and open complementary split ring resonators to the synthesis of artificial transmission lines and microwave passive components," IEEE Transactions on Microwave Theory and Techniques, vol. 57, no. 12, pp. 3395-3403, 2009.

[28] M. Durán-Sindreu, A. Vélez, G. Sisó et al., "Recent advances in metamaterial transmission lines based on split rings," Proceedings of the IEEE, vol. 99, no. 10, pp. 1701-1710, 2011.

[29] M. Durán-Sindreu, P. Vélez, J. Bonache, and F. Martín, "Broadband microwave filters based on Open Split Ring Resonators (OSRRs) and Open Complementary Split Ring Resonators (OCSRRs): improved models and design optimization," Radioengineering, vol. 20, no. 4, pp. 775-783, 2011.

[30] J. D. Baena, J. Bonache, F. Martín et al., "Equivalent-circuit models for split-ring resonators and complementary splitring resonators coupled to planar transmission lines," IEEE Transactions on Microwave Theory and Techniques, vol. 53, no. 4, pp. 1451-1460, 2005.

[31] J.-S. Hong and M. J. Lancaster, Microstrip Filters For RF/Microwave Applications, John Wiley \& Sons, New York, NY, USA, 2001. 

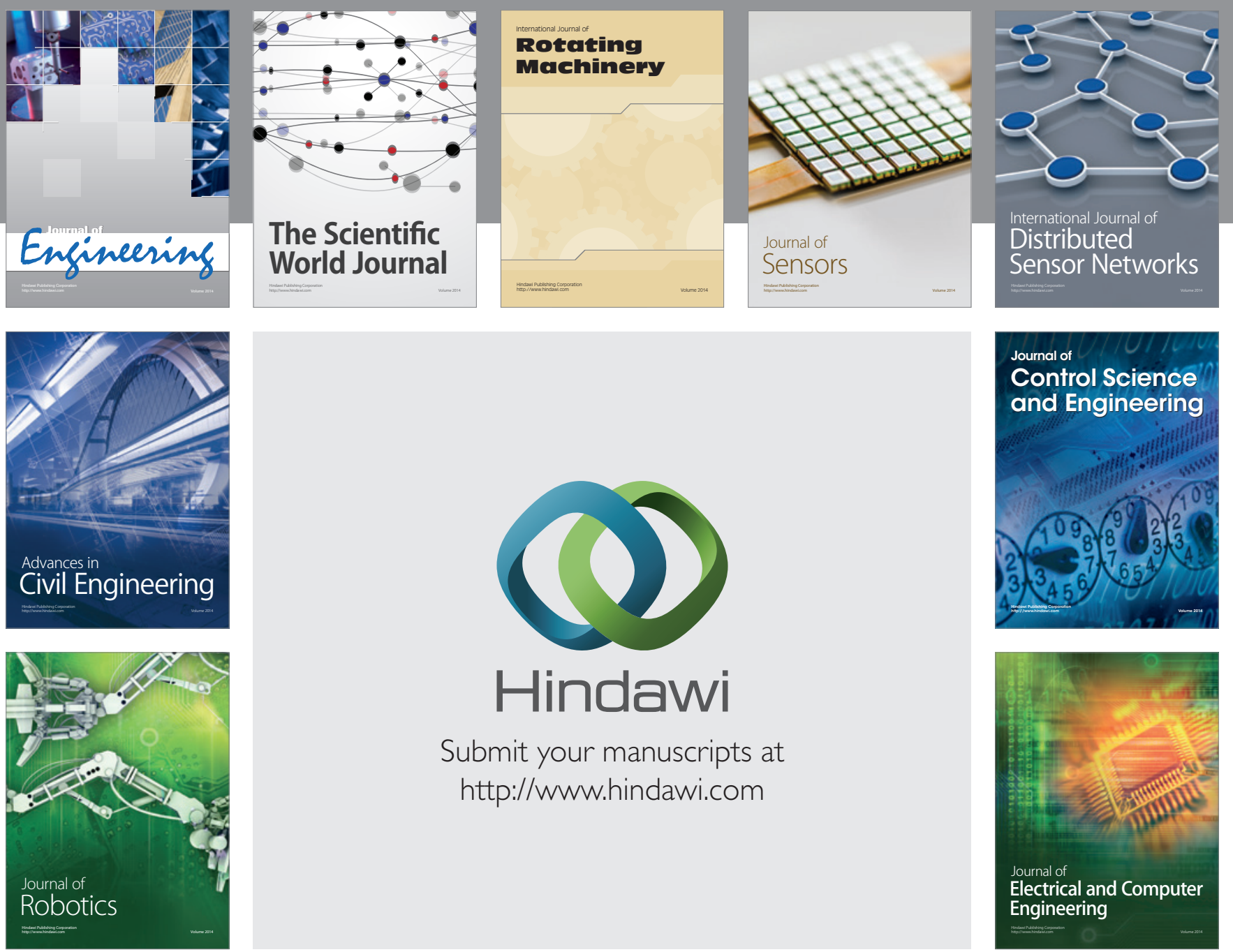

Submit your manuscripts at

http://www.hindawi.com
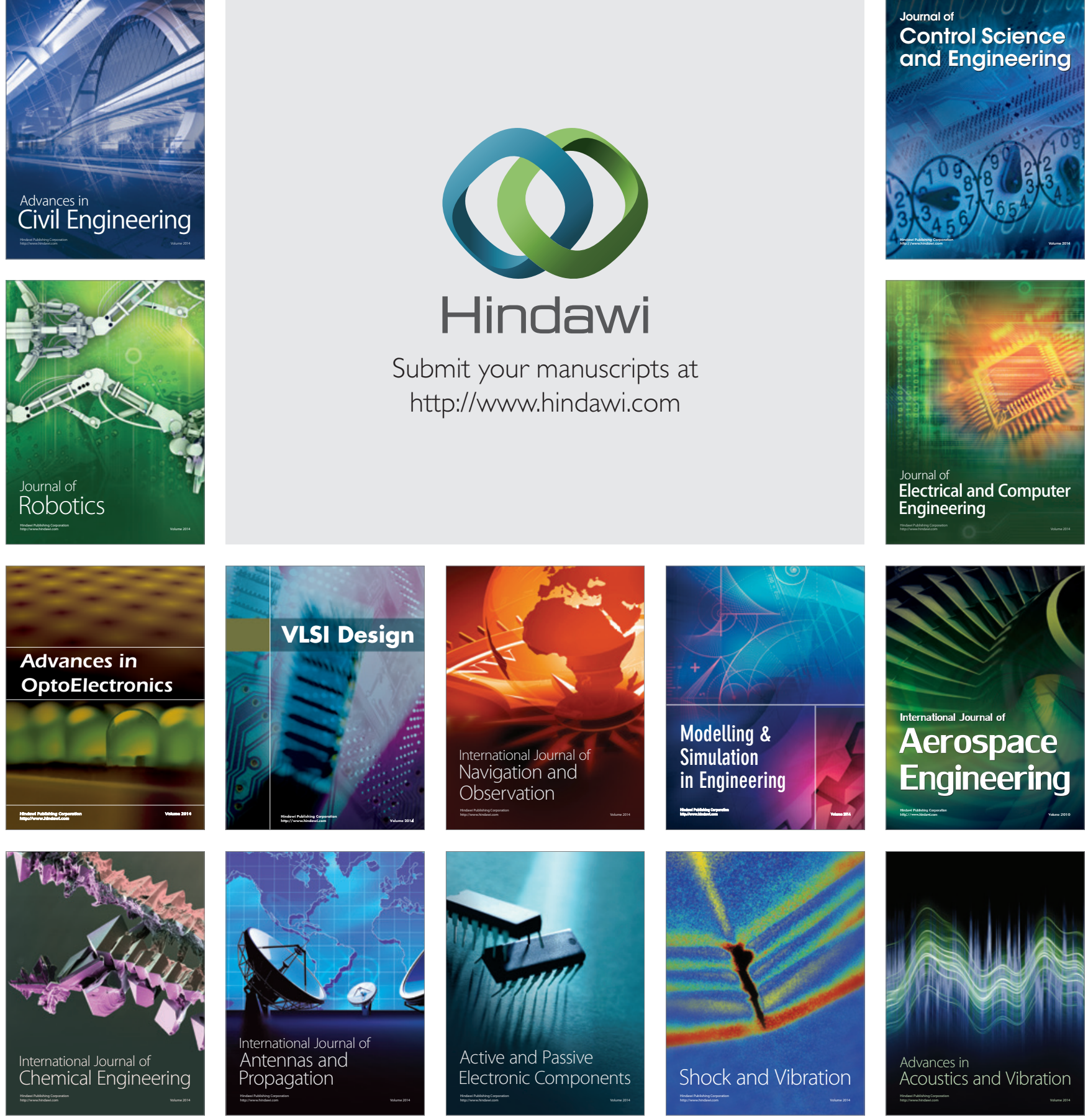